\title{
Diluting the material forming the second generation stars in globular clusters: the contribution by unevolved stars (Research Note)
}

\author{
R. G. Gratton ${ }^{1}$ and E. Carretta ${ }^{2}$ \\ 1 INAF-Osservatorio Astronomico di Padova, Vicolo dell'Osservatorio 5, 35122 Padova, Italy \\ e-mail: raffaele.gratton@oapd.inaf.it \\ 2 INAF-Osservatorio Astronomico di Bologna, via Ranzani 1, 40127 Bologna, Italy
}

Received 17 May 2010 / Accepted 14 July 2010

\section{ABSTRACT}

\begin{abstract}
In this short communication we consider the possibility that stars less evolved than the polluters are the source of the dilution needed to explain the observed composition of second-generation globular cluster (GC) stars and the $\mathrm{Na}-\mathrm{O}$ and $\mathrm{Mg}$ - $\mathrm{Al}$ anticorrelations. If these stars can lose $0.5-1 \%$ of their mass during the relevant epochs, there is enough diluting material to produce the observed anticorrelations. In this case, the original mass of proto-GCs was several tens times higher than the current mass of GCs. While not strictly impossible, this is a stringent hypothesis that needs more support. Should this scenario be found true, then the link between the primordial (first-generation) population in GC and the field population would be very strong.
\end{abstract}

Key words. stars: abundances - stars: atmospheres - stars: population II - globular clusters: general

\section{Introduction}

For the past few years, it has been known that at least two stellar generations are present in all globular clusters (GCs) (Gratton et al. 2001; Ramirez \& Cohen 2002; see Gratton et al. 2004, for a review). Extensive studies have shown that the second generation, characterized by overabundance of $\mathrm{Na}$ and sometimes $\mathrm{Al}$, and by a deficiency in $\mathrm{O}$ (that has been transformed into $\mathrm{N}$ ) is dominant, since it is responsible for about $2 / 3$ of the stars (Carretta et al. 2009a); the remaining $\sim 1 / 3$ of the stars have a composition that is virtually indistinguishable from that of field stars. The stars overabundant in $\mathrm{Na}$ and deficient in $\mathrm{O}$ are generally distributed over a range of values in $[\mathrm{Na} / \mathrm{Fe}]$ and $[\mathrm{O} / \mathrm{Fe}]$, describing an Na-O anticorrelation and a similar (but not identical) Al-Mg one (Kraft 1994; see Carretta et al. 2009a,b, for several examples). Prantzos \& Charbonnel (2006) show that these anticorrelations can be reproduced by assuming a typical polluter composition, which is then diluted by different amounts of pristine material. Such a dilution would also help explain the constraints from observed Li abundances, because significant amounts of this easily destroyed element appear to be present in most GC stars (Bonifacio et al. 2002; Pasquini et al. 2005; Lind et al. 2009; D'Orazi et al. 2010).

The observed homogeneity in heavy elements (Fe, $\alpha$ - and Fe-group species) in GCs and the very peculiar chemical composition of the polluters strongly suggest that they only belong to a limited range of masses, which marginally contributes to the overall galactic chemical enrichment. When combined with the large fraction of GC stars belonging to the second generation, this suggests that only a small fraction of the primordial population remains bound to the cluster and that the original episode of star formation was much larger (Prantzos \& Charbonnel 2006; Carretta et al. 2010).
While these facts have been assessed well, a number of important issues still remain open, precluding full understanding of the early phases of these massive clusters. They include (i) the nature of the polluters, which may be either massive asymptotic giant branch (AGB) stars undergoing hot bottom burning (Ventura et al. 2001) or fast-rotating massive stars (FRMS; Decressin et al. 2007), and other hypotheses also proposed (e.g. massive binaries, de Mink et al. 2009); (ii) the distribution of stars along the anticorrelation, which might be either continuous or clumped at some specific value (see e.g. the case of NGC 2808: D'Antona \& Caloi 2004; Carretta et al. 2006, etc.); and (iii) the origin of the diluting material. In this short paper, we focus our attention on this last issue.

D'Ercole et al. (2008) and Decressin et al. (2007) have considered scenarios for justifying this diluting material. According to Decressin et al. (2007), second-generation stars form in circumstellar disks around FRMS. Depending on the epoch when mass loss occurs, the polluted material is mixed with various amounts of unprocessed material previously expelled by the outer regions of the same massive stars, so that stars form with progressively higher fraction of polluting material. This scenario has serious difficulties in explaining clumpy distribution of stars along the $\mathrm{Na}-\mathrm{O}$ anticorrelation, such as those observed in NGC 2808. Furthermore, it is not clear how this scenario can generate a bound cluster.

On the other hand, D'Ercole et al. (2008) proposes that the slow winds from the massive AGB stars generate a cooling flow, creating a very dense cloud from which second-generation stars form. In their scenario, dilution is obtained by assuming that part of the original gas is left in a torus, which for some not entirely clear reasons is not polluted by the ejecta of the core collapse supernovae of the primordial population, and then after some time falls back onto the dense cloud that is forming the secondgeneration stars, hence stars with a progressively larger fraction 
of diluting material form. Both a clumpy distribution (see also D'Ercole et al. 2010) and a strongly bound cluster can be easily obtained. However, the hypothesis that a reservoir of pristine gas exists appears ad hoc, and somewhat unacceptable.

In this paper we explore an alternative origin for the reservoir of pristine gas, which combines concepts from both the Decressin et al. and D'Ercole et al. scenarios. We consider the possibility that the reservoir of pristine gas is produced by mass lost from less evolved stars (typically - but not only - main sequence stars) than those producing the polluting material. This hypothesis has several interesting features: (i) the mechanism is not ad hoc, since stars of lower mass than the polluters are surely losing some mass simultaneously to the more massive ones, which are in later evolutionary phases (actually, this mass should be incorporated into the hydrodynamical and chemical models); (ii) the material lost by these stars will still be unprocessed, but see discussion at the end of Sect. 4; (iii) we should not worry about the exact timing of the fallback of an unprocessed cloud; (iv) we do not expect this material to be significantly enriched by the core collapse supernovae of the primordial population. However, this hypothesis may work only if enough mass is lost by the lower mass stars at the relevant epoch, as discussed in the rest of this paper.

\section{The basic idea}

We need some material with original composition to dilute the polluted one, in order to (i) reproduce the observed run of $\mathrm{O}$ and $\mathrm{Na}$; and (ii) to have some $\mathrm{Li}$ saved. Evidence for this last point comes from NGC 6397, where Bonifacio et al. (2002) find $\log n(\mathrm{Li}) \sim 2.3$ in most stars, and from NGC 6752 where Pasquini et al. (2005) find in Na-rich/O-depleted stars $\log n(\mathrm{Li}) \sim 1.93$ for $[\mathrm{O} / \mathrm{Fe}]=-0.2 \mathrm{dex}$. In this hypothesis, $\mathrm{Li}$ should be depleted as $\mathrm{O}$.

We note that the dilution factor in GCs is in the range $0-0.7^{1}$ : this is the amount required to place on the Na-O anticorrelation the intermediate I stars, that represent the bulk of the cluster population (see Gratton et al. 2010; Carretta et al. 2009a). This means that the amount of primordial, diluting material is roughly equal to half the amount of processed material. However, to be conservative, we assumed that the diluting mass is similar to the polluting one throughout this paper.

How it is possible to achieve this? The explanation proposed by D'Ercole et al. (2008) is some primordial gas saved from pollution by core-collapse SNe. An alternative solution, explored in this note, is to consider the mass lost from the stars less evolved than the polluters.

Our working hypotheses are:

1. the stars distribute by number as $n(M)=M^{x}$. We assume a lower mass limit of $0.2 M_{\odot}$, and an upper limit of $100 M_{\odot}$. As an alternative, we use the Kroupa (2001) mass function (between 0.01 and $100 M_{\odot}$ );

2. the polluted material comes from stars with masses in the mass range $M_{\min }$ to $M=8 M_{\odot}$, and they lose all the mass except for that is left in compact remnants. This is assumed to be a linear function of the original mass of the star, which is $0.54 M_{\odot}$ for a star of $0.9 M_{\odot}$, and $1.24 M_{\odot}$ for a star of $8 M_{\odot}$ (see Ferrario et al. 2005);

3. The diluting primordial material comes from mass lost by all stars with masses less than $M_{\text {min }}$ (including low-mass stars).

\footnotetext{
${ }^{1}$ Stars with a dilution $\sim 1$ can essentially be identified with the primordial population.
}

Table 1. Fraction of mass $(\alpha)$ lost by less evolved stars assuming a maximum polluter mass of $8 M_{\odot}$.

\begin{tabular}{|c|c|c|c|c|}
\hline \multicolumn{5}{|c|}{$M_{\min }=4 M_{\odot}$} \\
\hline$x$ & $\begin{array}{l}\text { Mass fraction } \\
\text { in stars of } \\
0.2-4 M\end{array}$ & $\begin{array}{l}\text { Mass fraction } \\
\text { in stars of } \\
\text { of } 4-8 M\end{array}$ & $\alpha$ & $\begin{array}{c}\text { Original ratio } \\
\text { 1st gen./GC }\end{array}$ \\
\hline-2.9 & 0.948 & 0.025 & 0.0208 & 16.9 \\
\hline-2.6 & 0.875 & 0.050 & 0.045 & 8.5 \\
\hline-2.3 & 0.727 & 0.083 & 0.090 & 5.1 \\
\hline-2.0 & 0.502 & 0.108 & 0.170 & 3.9 \\
\hline Kroupa & 0.845 & 0.0053 & 0.00489 & 7.0 \\
\hline$x$ & $\begin{array}{l}\text { Mass fraction } \\
\text { in stars of } \\
0.2-5 M_{\odot}\end{array}$ & $\begin{array}{c}M_{\min }=5 M_{\odot} \\
\text { Mass fraction } \\
\text { in stars of } \\
\text { of 5-8 } M_{\odot}\end{array}$ & $\alpha$ & $\begin{array}{c}\text { Original ratio } \\
1 \text { st gen./GC }\end{array}$ \\
\hline-2.9 & 0.959 & 0.0146 & 0.0126 & 27.6 \\
\hline-2.6 & 0.895 & 0.030 & 0.0279 & 13.4 \\
\hline-2.3 & 0.758 & 0.052 & 0.057 & 7.7 \\
\hline-2.0 & 0.540 & 0.070 & 0.108 & 5.7 \\
\hline Kroupa & 0.864 & 0.035 & 0.0331 & 10.9 \\
\hline$x$ & $\begin{array}{l}\text { Mass fraction } \\
\text { in stars of } \\
0.2-6 M_{\odot}\end{array}$ & $\begin{array}{c}M_{\min }=6 M_{\odot} \\
\text { Mass fraction } \\
\text { in stars of } \\
\text { of } 6-8 M_{\odot}\end{array}$ & $\alpha$ & $\begin{array}{c}\text { Original ratio } \\
\text { 1st gen./GC }\end{array}$ \\
\hline-2.9 & 0.965 & 0.0086 & 0.0070 & 49.4 \\
\hline-2.6 & 0.912 & 0.0182 & 0.0158 & 23.3 \\
\hline-2.3 & 0.778 & 0.032 & 0.0328 & 13.1 \\
\hline-2.0 & 0.565 & 0.045 & 0.062 & 9.5 \\
\hline Kroupa & 0.878 & 0.021 & 0.0181 & 19.8 \\
\hline$x$ & $\begin{array}{l}\text { Mass fraction } \\
\text { in stars of } \\
0.2-7 M_{\odot}\end{array}$ & $\begin{array}{c}M_{\min }=7 M_{\odot} \\
\text { Mass fraction } \\
\text { in stars of } \\
\text { of } 7-8 M_{\odot}\end{array}$ & $\alpha$ & $\begin{array}{c}\text { Original ratio } \\
\text { 1st gen./GC }\end{array}$ \\
\hline-2.9 & 0.971 & 0.0037 & 0.0030 & 117.1 \\
\hline-2.6 & 0.917 & 0.0080 & 0.0065 & 55.6 \\
\hline-2.3 & 0.736 & 0.0147 & 0.0138 & 30.3 \\
\hline-2.0 & 0.589 & 0.0207 & 0.0262 & 21.5 \\
\hline Kroupa & 0.889 & 0.0102 & 0.00715 & 48.8 \\
\hline
\end{tabular}

\section{The toy model}

In our toy model we consider that all stars with masses less than $M_{\text {min }}$ lose a fraction $\alpha$ of their mass during the relevant phase. For this case, the exponent of the mass function $x$, the fraction of mass enclosed in stars in the different mass ranges, the values of $\alpha$ required to produce as much diluting as polluting mass, and the ratio between the mass in the primordial generation and in the $\mathrm{GC}^{2}$, are listed in Table 1.

The minimum mass required to produce the right amount of unpolluted material from stars is given roughly by the relation:

$M_{\min } \sim 15.8+3.7 \cdot x \quad M_{\odot}$

if $\alpha=0.01$. Assuming $x=-2.3$, the original ratio primordialto-GC is 40.6 and $M_{\min }=7.25$; for $x=-2.6$ and $x=-2.9$, the minimum masses are $M_{\min }=6.65$ and $M_{\min }=5.45$, and the ratios are 36.6 and 34.4 , respectively.

An exam of the values listed in Table 1 shows that the ratio between the original mass of the primordial population and that of the GC is roughly given by $\sim-0.97 /(x \alpha)$. Furthermore, to have the right amount of diluting material, $\alpha$ should be about proportional to the range of masses of the polluters: a wider range of masses requires a higher value of $\alpha$.

\footnotetext{
2 This is the mass of the GC after formation of the second generation. Current mass should be lower.
} 
Table 2. Fraction of mass $(\alpha)$ lost by less evolved stars assuming various ranges of polluter masses and either $x=-2.3$ or the Kroupa (2001) mass function).

\begin{tabular}{ccccc}
\hline \hline $\begin{array}{c}\text { Polluters } \\
\text { Mass range } \\
\left(M_{\odot}\right)\end{array}$ & $\begin{array}{c}\text { Mass fraction } \\
\text { in stars of } \\
0.2-M_{\min } M_{\odot}\end{array}$ & $\begin{array}{c}\text { in polluters } \\
\text { mass range }\end{array}$ & $\alpha$ & $\begin{array}{c}\text { Original } \\
\text { ratio } \\
\text { 1st gen/GC }\end{array}$ \\
\hline \multicolumn{5}{c}{$x=-2.3$} \\
$7-8$ & 0.795 & 0.0147 & 0.0138 & 30.3 \\
$6-7$ & 0.778 & 0.0169 & 0.0169 & 25.4 \\
$5-6$ & 0.756 & 0.0214 & 0.0214 & 20.7 \\
$4-5$ & 0.727 & 0.0288 & 0.0280 & 16.3 \\
\hline \multicolumn{5}{c}{ Kroupa mass function } \\
$7-8$ & 0.889 & 0.0102 & 0.00715 & 48.8 \\
$6-7$ & 0.878 & 0.0123 & 0.0109 & 36.2 \\
$5-6$ & 0.864 & 0.0153 & 0.0111 & 32.6 \\
$4-5$ & 0.845 & 0.0199 & 0.0150 & 24.9 \\
\hline
\end{tabular}

Table 3. Age, turn-off mass, $M_{\mathrm{TO}}$, and mass of AGB stars, $M_{\mathrm{AGB}}$.

\begin{tabular}{ccc}
\hline \hline Age & $M_{\mathrm{TO}}$ & $M_{\mathrm{AGB}}$ \\
\hline $3.85 \times 10^{7}$ & 7.70 & 8.00 \\
$6.3 \times 10^{7}$ & 6.07 & 6.34 \\
$1.0 \times 10^{8}$ & 4.85 & 5.10 \\
$2.0 \times 10^{8}$ & 3.47 & 3.68 \\
\hline
\end{tabular}

As an alternative case we may consider a given value of e.g. $x=-2.3$ (or the Kroupa mass function) and assume that the range of mass of the polluters is always equal to $1 M_{\odot}$. In this way we get the values of Table 2 . On the other hand, $\alpha$ represents the total mass lost during the relevant period. The length of this period depends on the range of mass under consideration. The mass loss rates required to produce the same value of $\alpha$ should then scale inversely proportional to this length.

We quantified the relevant data in Table 3, using the isochrones by Bertelli et al. (2008). The time required for the evolution of $5-8 M_{\odot}$ stars is from $3.85 \times 10^{7}$ to $1.1 \times 10^{8} \mathrm{yr}$ after the start of the formation of primordial generation stars, that is, the mass lost by less evolved stars over an interval of about 70 Myr may be used to dilute the polluting material. On the other hand, if the polluters were limited to the range of masses from 7.3 to $8 M_{\odot}$, the interval of time would be only $8 \mathrm{Myr}$. The same value of $\alpha$ would then require almost ten times higher mass loss rates. Since the length of the useful interval of time scales with $M_{\text {min }}$ more rapidly than $\alpha$, a higher mass loss rate is required to produce the required dilution if the range of mass is narrower. Alternatively, if the mass loss rate (for stars of the same mass) is the same for all GCs, we should expect less dilution (hence a more clumpy distribution of abundances along the anticorrelation) for those GCs with a more restricted mass range for polluters.

\section{Limits on $\alpha$}

There are upper limits to $\alpha$ from other observations and considerations; for example, $\alpha$ for solar type stars is limited because too much mass loss would not be compatible with both Li depletion in the pre-main sequence phase and observation of the Spite plateau, as well as with the dilution observed in normal Population II stars at the base of the subgiant branch.

Unfortunately, the mass loss rates are largely uncertain. For solar type stars, Wood et al. (2002) suggest $\dot{M} \sim$ $4 \times 10^{5} / t^{-2.00 \pm 0.52}$, but also a saturation at young ages (at
$2.5 \times 10^{-11} M_{\odot} / \mathrm{yr}$ ), with an uncertainty of a factor 5 . The best estimate of the mass lost during the epoch $40-110$ Myr is $2.0 \times$ $10^{-3} M_{\odot}$, but the upper limit is $10^{-2} M_{\odot}$. According to Holzwarth \& Jardine (2007), a value 100 times solar $\left(2 \times 10^{-14} M_{\odot} / \mathrm{yr}\right)$ is reasonable for solar type stars in this age range. This makes the total mass lost of $2 \times 10^{-12} \times 7 \times 10^{7} \sim 1.4 \times 10^{-4} M_{\odot}$ two orders of magnitudes too low over the relevant period. On the other hand, empirical evidence (Lednicka \& Stepien 2008) suggests an upper limit of $0.05 M_{\odot}$ for Praesepe $(0.7 \mathrm{Gyr})$. If this is lost at a constant rate, it would give $5 \times 10^{-2} / 7 \times 10^{8}$, which is $7 \times 10^{-11} M_{\odot} / \mathrm{yr}$. Integrated over $7 \times 10^{7} \mathrm{yr}$, this gives $5 \times 10^{-3} M_{\odot}$.

In turn, the Li-dip may be explained if $0.05 M_{\odot}$ is lost from $1.3 M_{\odot}$ (solar metallicity) stars (Schramm et al. 1990; see also Russell 1995). The proposed mechanism is related to the main instability strip, with a mass loss rate of $10^{-11} M_{\odot} /$ yr. Integrated over $70 \mathrm{Myr}$, this yields $7 \times 10^{-4} M_{\odot}$.

Summarizing, an upper limit to mass lost during the 40-110 Myr epoch for a solar type star is $0.01 M_{\odot}$. This implies a maximum mass loss rate of $\sim 10^{-10} M_{\odot} /$ yr. On the other hand, in our approach, $\alpha$ is a suitable average value for the fraction of mass lost during this phase by all stars that are less evolved than those causing the pollution. This average also includes (i) lowmass stars that are still in the pre-main sequence phase at the relevent epoch $\left(M<0.5 M_{\odot}\right.$ : Di Criscienzo et al. 2009) ${ }^{3}$; (ii) the same stars later providing the polluting material, but in earlier evolutionary phases (e.g. while they are in the Cepheids instability strip, see e.g. Neilson \& Lester 2009); and (iii) interacting binaries (see e.g. Mennickent et al. 2010). All these classes of objects may have mass-loss rates (in units of the stellar mass) that are significantly more than solar type stars of similar ages. The assumption of a value $\alpha \sim 1 \%$ therefore does not appear completely implausible.

It should, however, be noticed that the chemical composition of the winds from these other classes of stars might not precisely reflect the original composition. Low-mass stars should have burnt Li during the pre-main sequence phase, when they were still fully convective. If they contributed significantly to the dilution required to explain the $\mathrm{O}-\mathrm{Na}$ anticorrelation, then the Li observed in second-generation stars with a high degree of dilution should have been produced by the same polluters, and there should not be a simple Na-Li anticorrelation. As a matter of fact, this is not at all excluded by current observations (see e.g. D’Orazi \& Marino 2010). For the other classes of objects, Luck \& Lambert (1992) made a chemical abundance analysis of Cepheids in the LMC and SMC. The $[\mathrm{O} / \mathrm{Fe}],[\mathrm{Na} / \mathrm{Fe}],[\mathrm{Mg} / \mathrm{Fe}]$, and $[\mathrm{Al} / \mathrm{Fe}]$ ratios are roughly solar, so that a putative wind from these stars may contribute to the dilution needed to explain the $\mathrm{Na}-\mathrm{O}$ and $\mathrm{Mg}-\mathrm{Al}$ anticorrelations. However, these stars are depleted in $\mathrm{C}$ and $\mathrm{Li}$, with evidence of dredge-up of N-rich material. For interacting binaries, depletion of $\mathrm{C}$ and excesses of $\mathrm{N}$ have been found in most Algols (Parthasarathy et al. 1983; Tomkin et al. 1993), and even O is depleted in the more massive $\beta$ Lyr (Balachandran et al. 1986; indeed, massive binaries have been proposed as a source of the polluting material, rather than as diluters: de Mink et al. 2009). If these two classes of objects were indeed the source of the diluters, we should then expect that the $\mathrm{Na}-\mathrm{O}$ and the $\mathrm{C}-\mathrm{N}$ anticorrelations should appear quite

\footnotetext{
3 We notice that $\mathrm{T}$ Tau stars have disks with masses of $\sim 0.01 M_{\odot}$, which however are probably dissipated on a timescale of $\sim 6 \mathrm{Myr}$ (Hillenbrand et al. 1998; Haisch et al. 2001). The dissipation time might be longer for stars of lower mass and brown dwarfs (Carpenter et al. 2006; Apai et al. 2007).
} 
different. This prediction might be compared with the actual data (see e.g. Briley et al. 2004).

\section{Limits on the ratio primordial population/GC}

The ratio of primordial population/GC cannot be greater than the ratio between the mass of the halo and the mass in GCs, which is $\sim 100$. In the current framework, this provides a lower limit for $\alpha$, that is, $\alpha>-0.0097 / x$.

If we assume $\alpha=0.01$ and $x=-2.3$, we should expect a mass ratio of primordial population to the GC of $\sim 40$. In this framework, to produce a cluster with a current mass of $10^{6} M_{\odot}$ (like NGC 2808 or $47 \mathrm{Tuc}^{4}$ ), one should start with $\sim 4 \times 10^{7} M_{\odot}$ of gas with a very homogeneous composition $(\Delta[\mathrm{Fe} / \mathrm{H}] \ll 0.04 \mathrm{dex})$; possibly a value of $\sim 8 \times 10^{7} M_{\odot}$ for the cloud mass would be more reasonable, considering a reasonable $\sim 0.5$ star formation efficiency for the primordial (original) generation. Star formation for the primordial generation cannot have lasted very long, or else SNe would have either (i) stopped it or (ii) contributed to the nucleosynthesis. A reasonable upper limit is $10^{7} \mathrm{yr}$. Such a massive $\left(4 \times 10^{7} M_{\odot}\right.$, stellar mass $)$ star-forming region should be very luminous $\left(M_{V} \sim-18\right)$.

Almost all $(98.7 \%)$ of the original cluster population would have been lost from the expansion of the cluster after $\mathrm{SNe}$ explosion and mass loss. The second burst of star formation (the one producing the second generation) would then be a much smaller episode. With a mass of $5 \times 10^{5} M_{\odot}$ of stars produced, it should have a luminosity of $M_{V}=-14$ at peak. At this epoch, the fading original population should still have a luminosity of $M_{V}=-16$, dominating the compact central cluster of secondgeneration stars.

Assuming a ratio $M / L=2$, all galactic GCs summed up have a mass of $3.4 \times 10^{7} M_{\odot}$. The primordial population should then be $\sim 1.4 \times 10^{9} M_{\odot}$. This requires that roughly half of the halo/thick disk mass comes from GCs (see Gratton et al. 2010, in preparation, where we will discuss the metallicity distribution and the element-to-element abundance ratios of the primordial population, which are very similar to that of the halo-thick disk).

\section{Can the wind from main sequence stars contribute to the second generation?}

Winds from main sequence stars are quite fast, with typical velocities of a few hundred $\mathrm{km} \mathrm{s}^{-1}$ (Neugebauer 1994; Dupree 2005). This is much higher than the escape velocity from a protoGC. Could this wind be contributing to the second generation? To address this issue, we follow the same arguments considered by Smith (1999), who considered the case for dissipation of intracluster gas (lost at low speed by red giants) from the energy injection by the high-velocity wind from the main sequence. We should replace the wind by red giants with the much stronger winds of AGB stars. The specific mass-loss rate for massive AGB stars is about two orders of magnitude more than that for current red giants: $\alpha_{\mathrm{AGB}} \sim 3 \times 10^{17} \mathrm{~s}^{-1}$. On the other hand, the specific mass loss rate for the diluters should be comparable to that of the AGB stars, or else dilution would be negligible.

Neglecting cooling for the moment, the winds from the diluters are kept within the cluster if Eq. (8) of Smith (1999) holds. Assuming $\alpha_{\mathrm{MS}} / \alpha_{\mathrm{AGB}}=0.5$, this equation requires that the wind velocity from the polluter $v_{\mathrm{MS}}$ satisfies the relation $v_{\mathrm{MS}}<5.2 \sigma_{\text {los }}$, where $\sigma_{\text {los }}$ is the line of sight velocity spread of

${ }^{4}$ We neglect here the mass lost by the GC after its formation. the proto-GCs. Current values of $\sigma_{\text {los }}$ are $\leq 20 \mathrm{~km} \mathrm{~s}^{-1}$ for almost all GCs. It is likely that $\sigma_{\text {los }}$ could have been slightly higher for proto-GCs. We conclude that winds with velocities $<100 \mathrm{~km} \mathrm{~s}^{-1}$ were almost certainly kept within the clusters. These include potential polluters like red giants in those phases preceding the AGB, close binaries, and perhaps surviving disks around lowmass stars.

On the other hand, even faster winds could contribute to dilution if the input kinetic energy is dissipated by radiative cooling. This may happen if radiative cooling losses are greater than the heating due to thermalization of the diluters' winds. To estimate when this may occur, we use inequality (21) of Smith (1999), which must be solved simultaneously with his Eq. (10). In this inequality, we should consider the radiative cooling coefficient appropriate to the central temperature of the intracluster wind, which can be taken from Faulkner \& Freeman (1977), as done by Smith (1999). Assuming a protocluster (core) radius of $1 \mathrm{pc}$ and central density of $10^{5} M_{\odot} \mathrm{pc}^{-3}$ (which are typical values for present GCs), we find that cooling was efficient at the relevant epoch insofar $\alpha_{\mathrm{MS}} / \alpha_{\mathrm{AGB}} \leq 0.2$. This value should be taken only as an order of magnitude estimate, owing to the approximations made and because several quantities are not well known. For instance, it assumes a wind velocity from MS stars of $600 \mathrm{~km} \mathrm{~s}^{-1}$, and is valid for the radius and densities considered above. It is possible that proto-GCs were more compact $(\sim 0.5 \mathrm{pc})$ and much denser $\left(>10^{6} M_{\odot} \mathrm{pc}^{-3}\right)$ (Marks \& Kroupa 2010). If we use the values for the original radius and central density from these authors, we get typical upper limits of $\alpha_{\mathrm{MS}} / \alpha_{\mathrm{AGB}} \leq 0.6$, which is compatible with a significant dilution by winds from main sequence stars.

We conclude that the wind from stars that are less evolved than the polluters might have contributed to the diluting material. This is very likely if a substantial fraction of this wind is at velocity $<100 \mathrm{~km} \mathrm{~s}^{-1}$, but it is still possible even for faster winds, at velocities of a few hundred $\mathrm{km} \mathrm{s}^{-1}$.

\section{Conclusions}

Assuming that the fraction of mass lost is roughly independent of stellar mass, then mass loss during MS (or more in general during the relevant period by stars less evolved than the polluters) may provide the diluting mass only if $\sim 1 \%$ (within a factor of 2 ) of the mass is lost in the relevant phase and the ratio of masses between the primordial generation and the $\mathrm{GC}$ is greater than 40 (still within a factor of 2). This requires that a considerable fraction of the halo (at least half) originated in proto-GCs, and also that these proto-GCs lost about $>95 \%$ of their primordial generation stars. Although we do not have direct access to events that occurred a Hubble time ago, chemical abundances allow us to put strong constraints even on those early phases of GC evolution. It is currently well known that second-generation stars are formed from the ejecta of massive stars of the primordial generation (e.g. Gratton et al. 2001). From our ongoing FLAMES survey we also know the current proportions of primordial and second-generation stars in GCs. The latter represent the bulk of stars in GCs (about 2/3) whereas the primordial component is still present, but at a level of only a third of the current stellar population. Taken together, these two observations mean that a large fraction of stars of the primordial generation must necessarily be lost from GCs.

Theoretical considerations about star formation efficiency (e.g. Parmentier et al. 2008), violent relaxation (Lynden-Bell 1967) and gas expulsion (e.g. Baumgardt et al. 2008) predict that a huge mass loss occurs in early phases of the GC lifetimes. 
In Carretta et al. (2010) we explored the estimates of the initial mass of the primordial generation in GCs required to satisfy these two observational features. With suitable (and realistic) assumptions on the initial mass function of primordial and secondgeneration stars, mass ranges of the preferred polluters (either AGBs or FRMS), and initial-final mass relation we were able to show that a proto-GC should have lost $\sim 90 \%$ of its primordial stellar population. This is a value that is not very distant from the $>95 \%$ we derive in the present paper.

As mentioned in Sect. 2, we assumed that the diluting mass is equal to the polluting one. Actually, it might be half of this value, so relaxing these constraints a bit. We conclude that, on the whole, this scenario is then quite constraining and not entirely implausible. Of course, a better determination of the mass loss from young stars would be highly welcomed.

Acknowledgements. We thank the referee for her/his very interesting comments, as well as for reminding us of the very interesting paper by Smith (1999). We thank Franca D'Antona and Annibale D'Ercole for useful discussions and Valentina D'Orazi for a critical reading of the paper. This research has been funded by PRIN MIUR 20075TP5K9.

\section{References}

Apai, D., Luhman, K., \& Liu, M. C. 2007, ASP Conf. Ser., 384, 383 Balachandran, S., Lambert, D. L., Tomkin, J., \& Parthasarathy, M. 1986, MNRAS, 219, 479

Baumgardt, H., Kroupa, P., \& Parmentier, G. 2008, MNRAS, 384, 1231

Bertelli, G., Girardi, L., Marigo, P., \& Nasi, E. 2008, A\&A, 484, 815

Bonifacio, P., Pasquini, L., Spite, F., et al. 2002, A\&A, 390, 91

Briley, M. M., Harbeck, D., Smith, G. H., \& Grebel, E. K. 2004, AJ, 127, 1588

Carpenter, J. M., Mamajek, E. E., Hillenbrand, L. A., \& Meyer, M. R. 2006, ApJ, 651, L49

Carretta, E., Bragaglia, A., Gratton, R. G., et al. 2006, A\&A, 450, 523

Carretta, E., Bragaglia, A., Gratton, R. G., et al. 2009a, A\&A, 505, 117

Carretta, E., Bragaglia, A., Gratton, R. G., \& Lucatello, S. 2009b, A\&A, 505, 139

Carretta, E., Bragaglia, A., Gratton, R. G., et al. 2010, A\&A, 516, A55

D'Antona, F., \& Caloi, V. 2004, ApJ, 611, 871

Decressin, T., Meynet, G., Charbonnel, C., Prantzos, N., \& Ekstrom, S. 2007, A\&A, 464, 1029 de Mink, S. E., Pols, O. R., Langer, N., \& Izzard, R. G. 2009, A\&A, 507, L1 D'Ercole, A., Vesperini, E., D’Antona, F., McMillan, S. L. W., \& Recchi, S. 2008, MNRAS, 391, 825

D'Ercole, A., D’Antona, F., Ventura, P., Vesperini, E., \& McMillan, S. L. W. 2010, MNRAS, 407, 854

di Criscienzo, M., Ventura, P., \& D’Antona, F. 2009, A\&A, 496, 223

D’Orazi, V., \& Marino, A. F. 2010, ApJ, 716, L166

D’Orazi, V., Lucatello, S., Gratton, R. G., et al. 2010, ApJ, 713, 11

Dupree, A. K., \& Brickhouse, N. S. 2005, Protostars and Planets V, LPI Contribution, 1286, 8458

Faulkner, D. J., \& Freeman, K. C. 1977, ApJ, 211, 77

Ferrario, L., Wickramasinghe, D., Liebert, J., \& Williams, K. A. 2005, MNRAS, 361,1131

Gratton, R. G., Bonifacio, P., Bragaglia, A., et al. 2001, A\&A, 369, 87

Gratton, R. G., Sneden, C., \& Carretta, E. 2004, ARA\&A, 42, 385

Gratton, R. G., Carretta, E., Bragaglia, A., Lucatello, S., \& D’Orazi, V. 2010, A\&A, 517, A81

Haisch, K. E., Lada, E. A., \& Lada, C. J. 2001, ApJ, 553, L153

Hillenbrand, L. A., et al. 1998, AJ, 116, 181

Holzwarth, V., \& Jardine, M. 2007, A\&A, 463, 11

Kraft, R. P. 1994, PASP, 106, 553

Kroupa, P. 2001, MNRAS, 322, 231

Lednika, A., \& Stepien, K. 2008, AN, 3299, 359

Lind, K., Primas, F., Charbonnel, C., Grundahl, F., \& Asplund, M. 2009, A\&A, 503,545

Luck, R. E., \& Lambert, D. L. 1992, ApJS, 79, 303

Lynden-Bell, D. 1967, MNRAS, 136, 101

Marks, M., \& Kroupa, P. 2010, MNRAS, 406, 2000

Mennickent, R. E., Kolaczkowski, Z., Graczyk, D., \& Ojeda, J. 2010, MNRAS, 405, 1947

Neilson, H. R., \& Lester, J. B. 2009, ApJ, 690, 1829

Neugebauer, M. 1994, in Mass Supply and Flows in the Solar Corona, ed. B. Fleck, G. Noci, \& G. Poletto (Dordrecht: Kluwer), 319

Parmentier, G., Goodwin, S. P., Kroupa, P., \& Baumgardt, H. 2008, ApJ, 678, 347

Parthasarathy, M., Lambert, D. L., \& Tomkin, J. 1983, MNRAS, 203, 1063

Pasquini, L., Bonifacio, P., Molaro, P., et al. 2005, A\&A, 441, 549

Prantzos, N., \& Charbonnel, C. 2006, A\&A, 458, 135

Ramirez, S., \& Cohen, J. G. 2002, AJ, 123, 3277

Russell, S. C. 1995, ApJ, 451, 747

Schramm, D. N., Steigman, G., \& Dearborn, D. S. P. 1990, ApJ, 359, L55

Smith, G. 1999, PASP, 111, 980

Tomkin, J., Lambert, D. L., \& Lemke, M. 1993, MNRAS, 265, 581

Ventura, P., D’Antona, F., Mazzitelli, I., \& Gratton, R. 2001, ApJ, 550, L65

Wood, B. E., Muller, H.-R., Zank, G. P., \& Linsky, J. L. 2002, ApJ, 574, 412 